\title{
Validity of reported pain as a measure of clinical state in juvenile rheumatoid arthritis
}

\author{
CAROLINE K ROSS, ${ }^{12}$ JOHN V LAVIGNE, ${ }^{3}$ JENNIFER R HAYFORD, ${ }^{4}$ \\ ALAN R DYER, ${ }^{2}$ AND LAUREN M PACHMAN ${ }^{14}$
}

From the ${ }^{1}$ Department of Pediatrics and the ${ }^{2}$ Multipurpose Arthritis Center, Northwestern University Medical School; the ${ }^{3}$ Department of Child Psychiatry; and the ${ }^{4}$ Division of Immunology/Rheumatology, Children's Memorial Hospital, Illinois

SUMmarY Reported pain is one valid indicator of clinical state which should be used in the assessment and management of children with juvenile rheumatoid arthritis. Parents' reports of children's pain for 101 children with juvenile rheumatoid arthritis differed significantly by type of juvenile rheumatoid arthritis. When multiple regression was used separately for pauciarticular and polyarticular classifications of juvenile rheumatoid arthritis a measure of clinical state, which reflected joint activity, morning stiffness, and overall disease activity as rated by the doctor, had a significant and independent effect upon pain reported by parents. The child's age was not significantly related to the pain reported by parents. Children's pain reports did not differ significantly between older and younger children.

Although pain has been found to be reliably reported by children with juvenile rheumatoid arthritis aged as young as $5,{ }^{1}$ the validity of reported pain as an indicator of clinical state has been discounted. $^{1-5}$ Children with juvenile rheumatoid arthritis have been found to suffer less discomfort in affected joints than adults with rheumatoid arthritis ${ }^{2}$; mean pain scores were found to be quite low and scores were not significantly correlated with disease severity and activity. ${ }^{1}$ Additionally, no significant differences were reported for visual analogue scores for pain on movement or pain at rest between groups receiving placebo or prednisolone in a double blind study, though differences were significant for other outcomes such as joint activity, rating of disease activity by the doctor, and grip strength. ${ }^{3}$

Reported pain may reflect cognitive development rather than clinical state. Laaksonen and Laine ${ }^{2}$ and Scott et al ${ }^{1}$ suggested that a relation between maturation and increased pain reporting might explain the lower reported pain by children. Beales et al explained the greater reported pain of older children (12-17 years) as due to their more mature

Accepted for publication 28 February 1989

Correspondence to Dr Caroline K Ross, Arthritis Section, Northwestern University Medical School, 303 East Chicago Avenue, Chicago, Illinois 60614, USA. understanding of disease and concern for its consequences. $^{45}$

This paper examines the validity of reported pain in juvenile rheumatoid arthritis as an indicator of clinical state. The following hypotheses were tested: (a) reported pain will increase with the child's age and $(b)$ reported pain will be unrelated to clinical state. Children's pain reports were used to test the first hypothesis. Parent's ratings of children's pain were used to test the second hypothesis in order to include children under five years in the analysis.

\section{Patients and methods}

Visual analogue scale pain reports from parents of 101 children, aged $1.4-16.7$ years, with a definite diagnosis of juvenile rheumatoid arthritis ${ }^{6}$ were obtained. Complete data were also obtained from 43 children aged more than 5 years whose parents provided data. A visual analogue scale without divisions was used. An analysis of differences between samples of children who completed pain ratings and children who did not indicates that apart from age there were no differences in sociodemographic, laboratory, diagnostic, or clinical characteristics.

The type of juvenile rheumatoid arthritis was determined at six months after onset, using criteria 
established by Brewer et al,${ }^{6}$ as pauciarticular, four or fewer joints $(44 \%)$; polyarticular rheumatoid factor positive, five or more joints $(2 \%)$; polyarticular rheumatoid factor negative $(35 \%)$; systemic $(9 \%)$; systemic onset with polyarticular involvement $(11 \%)$. Children classified as systemic had no observable arthritis. For analyses involving juvenile rheumatoid arthritis classification the five classifications were combined as follows: polyarticular (polyarticular rheumatoid factor negative and positive, systemic onset polyarticular), pauciarticular, and systemic. Owing to the small sample (nine patients), children with systemic juvenile rheumatoid arthritis were excluded from most analyses. The sample included 24 boys and 77 girls. Subjects were further classified into early onset-that is, before 6 years of age ( 55 girls, 14 boys) and late onset -6 years or later ( 22 girls, 10 boys). Thirteen girls with onset before 6 years had either active uveitis or a history of uveitis. Of the 101 children, 21 were antinuclear antibody positive (end point titre $\geqslant 1 / 80$ dilution on $\mathrm{HEp}_{2}$ or mouse stomach and kidney substrate (Kallestad Laboratories, Austin, TX) at time of enrolment.

Clinical state was assessed using estimates of joint inflammation based on the doctor's rating (1-4 scale based upon swelling, warmth, tenderness: none, mild, moderate, severe); child/parent report of morning stiffness (less than five minutes, five minutes to two hours, two hours or more); doctor's rating of disease activity at visit (inactive (less than 3 months) or questionably active, minimal, moderate, severe).

\section{Results}

Parent visual analogue scale reports of pain ranged from $0=$ no pain to $10=$ severe continuous pain. Mean pain ratings were $3.9 \mathrm{~cm}$, and $31 \%$ of parents reported ratings above the $5 \cdot 0$ scale midpoint. Child visual analogue scale ratings (from 43 children) ranged from 0 to 10 with a mean of 4.2 and $37 \%$ reporting above the midpoint.

A Pearson correlation of 0.35 for the 43 matched parent and child pain reports was significant $p=0.01$. Of the 43 paired ratings, $18(42 \%)$ differed by less than $1 \mathrm{~cm} ; 25(58 \%)$ differed by more than $1 \mathrm{~cm}$. In 12 of the 25 which differed by more than $1 \mathrm{~cm}$, the child rated pain as more severe than that indicated by the parent and in 13 the parent rated the pain more severely than the child.

RELATION BETWEEN CHILDREN'S AGE AND REPORTED PAIN

For 17 children with pauciarticular juvenile rheumatoid arthritis the mean reported pain scores for younger children $(<12$ years) were $4 \cdot 2($ SD $2 \cdot 9) \mathrm{cm}$ compared with $2.4(3.3) \mathrm{cm}$ for older childre ( $>12$ years) with a $t$ value of $1 \cdot 14$. For 23 childrep. with polyarticular juvenile rheumatoid arthritis the mean scores for younger children $(<12$ years) wer $\overrightarrow{\bar{\theta}}$ $4.7(2.9) \mathrm{cm}$ compared with $4.9(3.6) \mathrm{cm}$ for oldew children $\left(>12\right.$ years) with a $t$ value of $-0 \cdot 1 \frac{5}{5}$ Neither comparison was statistically significant.

RELATION BETWEEN REPORTED PAIN AND CLINICAL STATE

When analysis of variance was used the mean paren $\vec{\oplus}$ pain ratings were significantly different for the thre $\overrightarrow{\mathrm{e}_{\mathrm{t}}}$ classifications of juvenile rheumatoid arthritis $\mathrm{F}=15 \cdot 67, \mathrm{p}<0.001$. Mean pain scores were $2 \cdot 1$ fơ pauciarticular, 5.0 for polyarticular, and 1.8 for systemic juvenile rheumatoid arthritis.

A clinical state variable was created for each chil进 by combining scores, additively and withoup weighting, of joint inflammation, disease activity $\vec{\sim}$ and morning stiffness. Spearman correlations between the clinical state variable and parent pain report were 0.40 and 0.56 for pauciarticular an£ polyarticular respectively compared with correlations of 0.32 to 0.50 for the individual indicators.

Regression analysis was carried out for pauciarticular and polyarticular juvenile rheumatoid aụ̂ ritis. In both analyses the clinical state has

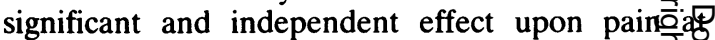
$\mathrm{p}<0.005$ (one tailed test) with $\beta=0.445$ for $\mathrm{p}$ ) articular juvenile rheumatoid arthritis and $\beta=0.41$ 호 for pauciarticular juvenile rheumatoid arthritis. Th8 effect of age was insignificant in both analyse $\beta=-0.078$ for polyarticular and $\beta=0.167$ for pauc articular juvenile rheumatoid arthritis.

\section{Discussion}

The data from this study do not support the्ष hypothesis that pain reports increase with the child age. Instead, these data suggest that reported pain one valid indicator of clinical state which may be used in the assessment and management of juvenile rheumatoid arthritis. For children with pauciarticula? juvenile rheumatoid arthritis the findings are the reverse of that suggested: younger children repoㅍ. greater pain than older children, not less. When this analysis of reported pain examined clinical state in conjunction with age it was the clinical state, nळळ age, that accounted for much of the variance in parent reported pain.

Parents' pain reports were used in the analysis of clinical state and reported pain in order to include the youngest children, from whom reports of pan cannot reliably be obtained using a visual analogue scale. 'Previous studies have used doctors' observif tions of pain behaviour upon examination ${ }^{2}$ and 
children's visual analogue score ratings of pain. ${ }^{134}$ Although some studies have suggested that parents may over-report or encourage their children to overreport pain, ${ }^{3}$ our data indicate that parental influences upon reporting, if they exist, are not systematic; children were as likely to report less pain than the parent as to report more pain.

It is important to know how much pain a child experiences with their disease. When a child suffers from a chronic illness in which pain is a predominant symptom the effects may be profound. Pain may limit physical function, school attendance, and social interaction, thereby interfering with the child's ability to learn and develop normally. The presence or absence of pain and the potential for diminished psychological or physical functioning has not yet been studied in juvenile rheumatoid arthritis. There have been no studies of the effects of painful juvenile rheumatoid arthritis in childhood upon social, psychological, and physical function in adulthood. Investigations of these issues could make a significant contribution to our understanding of psychological and social adjustment in juvenile rheumatoid arthritis and to our ability to assist children in achieving maximum functioning in adulthood.

Supported by grant NIH NIAMSD, Illinois Chapter Arthritis Foundation.

\section{References}

1 Scott R J, Ansell B M, Huskisson E C. Measurement of pain in juvenile chronic arthritis. Ann Rheum Dis 1977; 36: 186-7.

2 Laaksonen A L, Laine V. A comparative study of joint pain in adult and juvenile rheumatoid arthritis. Ann Rheum Dis 1961; 20: 386-7.

3 Kvien T K, Høyeraal H M, Sandstad B. Assessment methods of disease activity in juvenile rheumatoid arthritis-evaluated in a prednisolone/placebo double-blind study. J Rheumatol 1982; 9: 696-702.

4 Beales J G, Keen J H, Holt P J L. The child's perception of the disease and the experience of pain in juvenile chronic arthritis. $J$ Rheumatol 1983; 10: 61-5.

5 Beales J G, Holt P J L, Keen J H, Mellor V P. Children with juvenile chronic arthritis: their beliefs about their illness and therapy. Ann Rheum Dis 1983; 42: 481-6.

6 Brewer E J, Bass J, Baum J, et al. Current proposed revision of JRA criteria. Arthritis Rheum 1977; 20: 195. 